\title{
Total Body Water Dynamics Estimated with Bioelectrical Impedance Vector Analysis and B-Type Natriuretic Peptide After Exposure to Hypobaric Hypoxia: A Field Study
}

\author{
Giacomo Strapazzon, ${ }^{1}$ Matiram Pun, ${ }^{1}$ Tomas Dal Cappello, ${ }^{1}$ Emily Procter, ${ }^{1}$ \\ Piergiorgio Lochner, ${ }^{2}$ Hermann Brugger, ${ }^{1}$ and Antonio Piccoli ${ }^{3}$
}

\begin{abstract}
Strapazzon, Giacomo, Matiram Pun, Tomas Dal Cappello, Emily Procter, Piergiorgio Lochner, Hermann Brugger, and Antonio Piccoli. Total body water dynamics estimated with bioelectrical impedance vector analysis and B-type natriuretic peptide after exposure to hypobaric hypoxia: A field study. High Alt Med Biol. 18:384-391, 2017.The relationship between total body water (TBW) dynamics and N-terminal pro-B-type natriuretic peptide (NTproBNP), a stable metabolite of B-type natriuretic peptide, during acute high altitude exposure is not known. To investigate this, we transported 19 healthy lowland subjects to $3830 \mathrm{~m}$ with a helicopter after baseline measurements $(262 \mathrm{~m})$. The physiological measurements and clinical assessments were taken at 9, 24, 48, and 72 hours and on the eighth day of altitude exposure. A bioelectrical impedance vector analysis (BIVA) from height corrected Resistance-Reactance (R-Xc graph) was used to estimate TBW status. NT-proBNP was measured from venous blood samples. The changes in impedance vector were lengthened at $9(p=0.011), 48(p=0.033)$, and 72 hours $(p=0.015)$ indicating dehydration compared to baseline. However, there was no dehydration at 24 hours $(p>0.05)$ from the baseline and the subjects trended to get euhydrated from 9 to 24 hours $(p=0.097)$. The maximum percent changes in vector length from the baseline were within 10\%-15\%. There was a significant increase of natural $\operatorname{logarithm}(\ln )(\mathrm{NT}$-proBNP) after ascent with a peak at 24 hours, although similarly to BIVA values, $\ln (\mathrm{NT}$-proBNP) returned to baseline after 8 days of altitude exposure. The changes in impedance vector length were not correlated with the changes in $\ln (\mathrm{NT}-$ proBNP) $(\mathrm{r}=-0.101, p=0.656)$. In conclusion, the dehydration at high altitude as reflected by $10 \%-15 \%$ vector lengthening falls within "appropriate dehydration" in healthy lowland subjects. NTproBNP does not simply reflect the TBW status during acute high altitude exposure and needs further investigation.
\end{abstract}

Keywords: acclimatization; acute mountain sickness; bioelectrical impedance analysis; high altitude; NT-proBNP; phase angle; vector length

\section{Introduction}

$\mathbf{T}$ HE DECREASE IN PARTIAL PRESSURE of oxygen with increasing altitude triggers a number of physiological responses among lowlanders (West, 2004). Within hours of arrival at high altitude, physiological responses such as an increase in heart rate (HR), respiratory rate (RR), and diuresis occur. Hypoxic ventilatory response (HVR) quickly leads to respiratory alkalosis and the kidneys directly respond with an augmented diuresis to correct the increase in blood $\mathrm{pH}$ (Leaf and Goldfarb, 2007).

Clinically, dehydration is considered a risk factor for acute mountain sickness (AMS) (Norris et al., 2012), especially if associated with the physical exertion (Richardson et al.,

${ }^{1}$ EURAC Institute of Mountain Emergency Medicine, Bolzano, Italy.

${ }^{2}$ Department of Neurology, Saarland University Medical Center, Homburg, Germany.

${ }^{3}$ Department of Medicine DIMED, University Hospital of Padova, Padova, Italy.

(C) Giacomo Strapazzon et al., 2017; Published by Mary Ann Liebert, Inc. This article is available under the Creative Commons License CCBY-NC (http://creativecommons.org/licenses/by-nc/4.0). This license permits non-commercial use, distribution and reproduction in any medium, provided the original work is properly cited. Permission only needs to be obtained for commercial use and can be done via RightsLink. 
2009a, 2009b; Castellani et al., 2010), although findings from normobaric hypoxic chamber exposure do not seem consistent with this finding (Gatterer et al., 2013). Studies from the field suggest that fluid intake is associated with decreased incidence of AMS (Basnyat et al., 1999; Nerin et al., 2006). However, the definitive quantification of total body water (TBW) dynamics over the period of hours to days in healthy subjects exposed to hypobaric hypoxia remains unknown.

Benign forms of peripheral edema (i.e., swelling in limbs and face) are a common feature at altitude and usually resolve immediately on descent. This nonspecific edema could be interpreted as fluid retention linked to the pathogenesis of altitude illnesses (Hackett and Rennie, 1979; Hackett et al., 1981), although there has never been a systematic investigation to clarify this association. Bioelectrical impedance analysis (BIA) using the bioelectrical impedance vector analysis (BIVA) method is a convenient and may be more accurate method to estimate overall TBW status (Piccoli et al., 1994, 1996; Lukaski and Piccoli, 2012) than BIA alone in some circumstances (Fulco et al., 1992). BIVA has been successfully used to monitor body water status in different clinical settings such as renal failure patients (Piccoli, 2010), obese subjects (Buffa et al., 2013), athletes (Pollastri et al., 2016), and also during high altitude sojourns (Piccoli et al., 1996).

The B-type natriuretic peptide (BNP), produced by the ventricles of the heart, physiologically regulates vasodilation, diuresis, sodium excretion, inhibition of renin secretion, and suppression of aldosterone synthesis (Weber and Hamm, 2006). The BNP is elevated during high altitude exposure and is associated with increased AMS incidence (Feddersen et al., 2009; Woods et al., 2011a; Mellor et al., 2014) and severity, with an apparent net gain in TBW based on BIA measurements (Woods et al., 2012). However, the association of BNP with TBW dynamics at high altitude has not been monitored using the BIVA method.

Therefore, we implemented the BIVA technique to monitor TBW status during an acute, passive rapid ascent to high altitude and after an extended length of altitude exposure of up to 8 days in total. In addition, we also explored the association between BIVA and N-terminal pro-B-type natriuretic peptide (NT-proBNP, a stable metabolite of BNP), plus associated biochemical analyses derived from venous blood and urine samples.

\section{Methods}

\section{Study subjects and high altitude exposure}

A total of 19 healthy Caucasians were recruited. Subjects were excluded if they were $<18$ years of age or had acute or chronic cardiovascular, pulmonary, or renal diseases. The subjects were instructed to avoid any medications that would affect study outcome (i.e., acetazolamide and steroids). However, if subjects needed any medication during the study, the study physician was consulted and treatment was recorded. Subjects requiring additional treatment with nonsteroidal antiinflammatory agents were included into the study protocol.

Subjects adhered to a standardized food and fluid intake regime with regular meals. Additional fluid intake was ad libitum during the study period, and total fluid intake was monitored using a questionnaire. The mean daily fluid intake reported was $\sim 2 \mathrm{~L} /$ day during the high altitude stay.

In this study, the preacclimatization (recent altitude exposure) was defined as a stay of several days at $\geq 3000 \mathrm{~m}$ and/ or day tours $\geq 3000 \mathrm{~m}$ in the 2 months before the study.

Complete data were obtained for only 11 subjects because of logistical challenges and exclusion criteria for collected data, as illustrated in Figure 1.

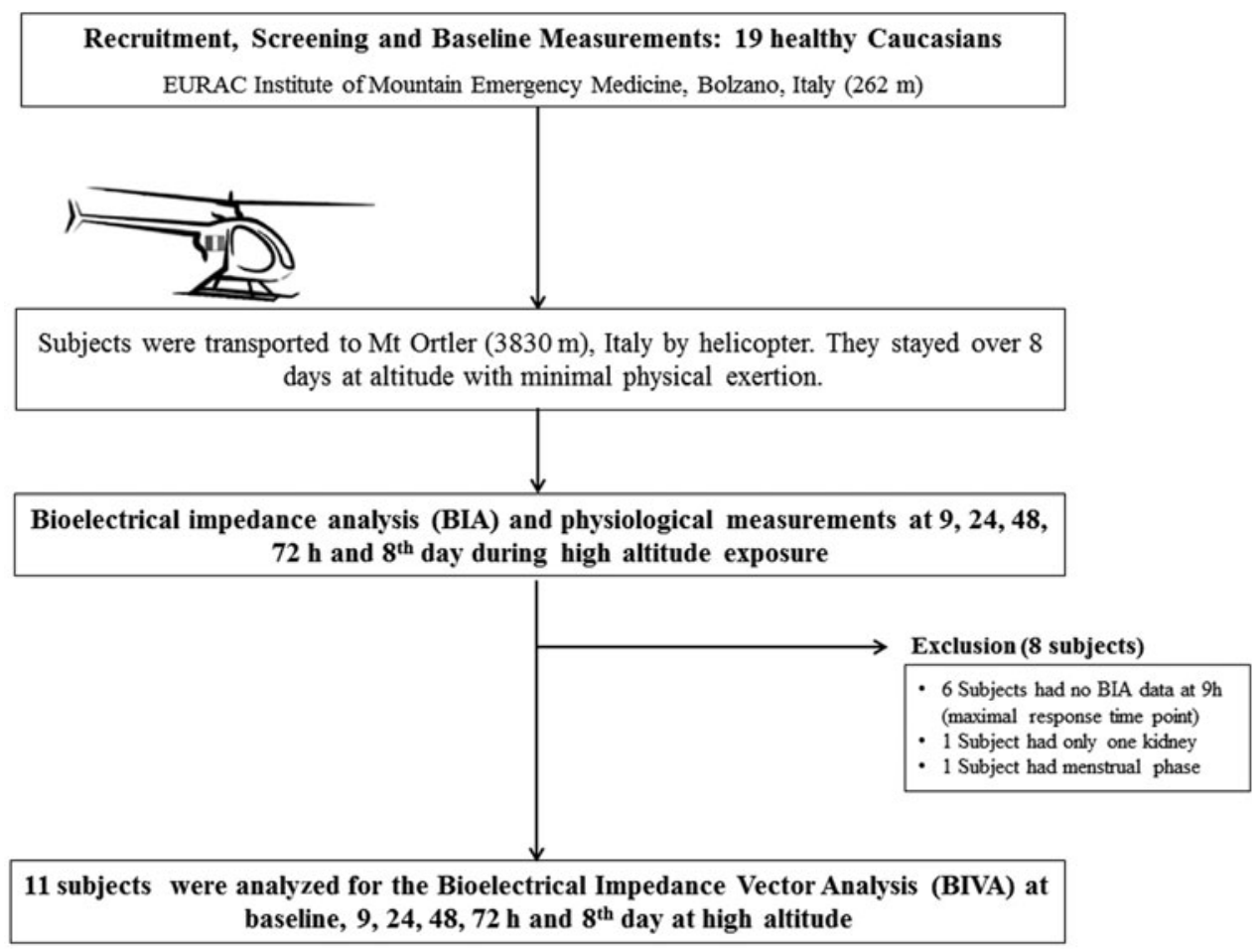

FIG. 1. Study design and data collection. Body water dynamics during acute acclimatization phase to $3830 \mathrm{~m}$ was analyzed for 11 subjects. 
The study was approved by the Ethics Committee of Bolzano General Hospital (number 0073450-BZ) and was conformed according to the Declaration of Helsinki (2008) (World Medical Association, 2013). Written informed consent was obtained from all subjects. The study was registered in the Clinical Trail Registry (www.clinicaltrials.gov, Trial Registration No.: NCT01794130).

\section{Data collection}

Subjects underwent a screening examination and baseline measurement at the Eurac Research Institute of Mountain Emergency Medicine, Bolzano, Italy (altitude $262 \mathrm{~m}$; barometric pressure $738 \mathrm{mmHg}$; oxygen partial pressure $143.16 \mathrm{mmHg}$ ). The subjects were transported by helicopter to the Mt. Ortler study site (altitude $3830 \mathrm{~m}$; barometric pressure $485 \mathrm{mmHg}$; oxygen partial pressure $102 \mathrm{mmHg}$ ), in the Italian Alps where they spent 8 days without undue physical exertion. The clinical examination and physiological measurements were taken at 9 , 24,48 , and 72 hours and on the eighth day in a warm medical tent at a controlled temperature at the study site as described elsewhere, to reproduce baseline conditions (Strapazzon et al., 2014, 2015). The clinical examination and physiological measurements included BIA, oxygen saturation $\left(\mathrm{SpO}_{2}\right.$; Handheld Pulse Oximeter NPB-40, Nellcor Puritan Bennett, Inc., Pleasanton, CA), HR, breathing rate, and Lake Louise Score (LLS). Venous blood and urine samples were taken at 9, 24, and 72 hours and on the eighth day. On the Mt. Ortler glacier, samples were collected every morning after sleep (apart from those after 9 hours).

\section{BIA and BIVA measurements}

BIA was completed with an impedance plethysmograph that emits an $800 \mu \mathrm{A}, 50 \mathrm{kHz}$ alternating current (BIA-101 Anniversary, AKERN SRL, Firenze, Italy). Measurements were taken with subjects in a supine position, post 15 minutes of rest. Surface contact electrodes (BIATRODES; Akern, Florence, Italy) were placed on the right hand and foot following manufacturer's guidelines. The BIA measurement yielded Resistance (R), Reactance (Xc), and Phase Angle. The BIA data on body water status were analyzed according to the BIVA method (Piccoli et al., 1994; Piccoli, 2010). The bivariate analysis of height corrected Resistance $(\mathrm{R} / \mathrm{H})$ and Reactance $(\mathrm{Xc} / \mathrm{H})$ gives rise to the $\mathrm{R}-\mathrm{Xc}$ graph in which an impedance vector reflects overall body water balance, without any assumption of body composition (Piccoli et al., 1996). The direction and length of the impedance vector in the bivariate plane of the $\mathrm{R}-\mathrm{Xc}$ graph are considered a reliable estimation of body water status, also at high altitude (Piccoli et al., 1994, 1996; Piccoli, 2010). The changes in the vector length at different time points of high altitude exposure are reported with corresponding $95 \%$ confidence ellipses.

\section{NT-proBNP and other biochemical parameters}

The biochemical parameters NT-proBNP, urea, creatinine, sodium $\left(\mathrm{Na}^{+}\right)$, potassium $\left(\mathrm{K}^{+}\right)$, chloride $\left(\mathrm{Cl}^{-}\right)$, and osmolality were analyzed from venous blood and urine samples. Venous blood samples were collected in serum/plasma tubes, centrifuged, and separated. Urine samples were collected in a sterile container provided to the subjects. All samples were stored in multiple aliquots at $-80^{\circ} \mathrm{C}$ until assayed. The NTproBNP was assayed using a VIDAS NT-proBNP2 Test Kit (bioMerieux SA, Marcy l'Etoile, France). The test kits are enzyme linked fluorescent assays (ELFA immunoassay) using monoclonal antibodies VIDAS. The VIDAS NTproBNP2 assay has a linearity from 15 to $25,000 \mathrm{pg} / \mathrm{mL}$ with a value $>125 \mathrm{pg} / \mathrm{mL}$ indicative of congestive cardiac failure. The mean coefficient of variation is $6 \%$ for intra- and $3 \%$ for interassay precision.

\section{Statistical analysis}

Data analysis for the BIVA measurements was carried out on 11 subjects as illustrated in Figure 1 (a number comparable to previous studies) (Piccoli et al., 1996; Ermolao et al., 2011). A reported value of $<20 \mathrm{pg} / \mathrm{mL}$ for NT-proBNP was considered $10 \mathrm{pg} / \mathrm{mL}$ for the calculations, due to assay precision limitations. To reduce variability, NT-proBNP values were transformed using a natural logarithm (ln). Vector length was calculated as the hypotenuse of individual impedance values (Lukaski et al., 2007). Analysis of variance (ANOVA) for repeated measures was used to analyze changes over time of study variables and biochemical parameters until 72 hours because data were available for only six subjects on the eighth day. Comparison of two time points was carried out by means of paired samples $t$-test. As the LLS was not normally distributed, the Friedman test was used instead of repeated measures ANOVA, plus Wilcoxon signed rank test was used instead of paired samples $t$-test. Similarly, multivariate ANOVA for repeated measures with Wilks' Lambda test was used to analyze changes over time in the impedance vector until 72 hours, plus paired one sample Hotelling's $\mathrm{T}^{2}$ test to calculate significant vector displacements. Holm-Bonferroni post hoc corrections were applied to all pairwise comparisons. A correlation coefficient for repeated observations (Bland and Altman, 1995) was used to detect if changes in NT-proBNP and vector length were correlated with changes in other parameters up until 72 hours. One of the two female subjects had extremely high NT-proBNP values without clinically relevant signs and symptoms and was excluded from the correlation analysis; the correlation analysis was carried out using data from 10 subjects. The statistical software SPSS version 23.0 (IBM Corp., Armonk, NY) was used for all statistical analyses, and $p<0.05$ was considered statistically significant. Values in each table are reported as mean \pm standard deviation unless stated otherwise.

\section{Results}

In total, 2 of the 11 subjects were female and 6 subjects reported preacclimatization. Subject characteristics are reported in Table 1. BIVA measurements (mean values), blood

Table 1. Subject Characteristics

\begin{tabular}{lc}
\hline Variable & Mean \pm SD or $\mathrm{n}(\%)$ \\
\hline Subjects & 11 \\
Age, years & $37 \pm 9$ \\
Male, $n(\%)$ & $9(82)$ \\
Height, cm & $175 \pm 8$ \\
Weight, kg & $73 \pm 9$ \\
BMI, kg/m & $23.6 \pm 1.8$ \\
Body fat, \% & $22.0 \pm 3.6$ \\
Altitude of residence, m & $530 \pm 400$ \\
Preacclimatization, $n(\%)$ & $6(55)$ \\
\hline
\end{tabular}

BMI, body mass index; f, female; $\mathrm{m}$, male; $n$, number; $\mathrm{SD}$, standard deviation. 
Table 2. Bioimpedance, Blood Pressure, Heart Rate, $\mathrm{SPO}_{2}$, Lake Louise Score, and Respiratory Variables

\begin{tabular}{|c|c|c|c|c|c|c|c|}
\hline Study variables & Baseline & 9 hours & 24 hours & 48 hours & 72 hours & 8 days & $\begin{array}{c}\text { Repeated } \\
\text { measures ANOVA } \\
\text { p-value }\end{array}$ \\
\hline $\mathrm{R} / \mathrm{H}(\Omega / \mathrm{m})$ & $290.5 \pm 24.3$ & $323.8 \pm 26.7$ & $299.6 \pm 27.6$ & $320.8 \pm 32.0$ & $321.1 \pm 34.5$ & $305.5 \pm 36.7$ & $<0.001$ \\
\hline $\mathrm{Xc} / \mathrm{H}(\Omega / \mathrm{m})$ & $36.7 \pm 3.7$ & $42.8 \pm 4.8$ & $38.2 \pm 7.0$ & $42.8 \pm 5.1$ & $44.7 \pm 5.4$ & $40.7 \pm 7.0$ & $<0.001$ \\
\hline Vector length $(\Omega / \mathrm{m})$ & $292.9 \pm 24.3$ & $326.7 \pm 26.8$ & $302.1 \pm 27.7$ & $323.7 \pm 32.3$ & $324.2 \pm 34.6$ & $308.3 \pm 37.1$ & $<0.001$ \\
\hline$\Delta \mathrm{VL}(\%)$ & - & $11.8 \pm 7.8$ & $3.3 \pm 6.2$ & $10.6 \pm 11.4$ & $10.7 \pm 8.9$ & $4.2 \pm 6.6$ & 0.007 \\
\hline Phase angle $\left(^{\circ}\right)$ & $7.1 \pm 0.8$ & $7.5 \pm 0.8$ & $7.0 \pm 1.2$ & $7.6 \pm 0.5$ & $7.8 \pm 0.8$ & $7.5 \pm 0.8$ & 0.028 \\
\hline $\mathrm{SBP}(\mathrm{mmHg})$ & $124.5 \pm 12.6$ & $131.5 \pm 14.1$ & $126.9 \pm 13.2$ & $126.6 \pm 13.5$ & $131.0 \pm 11.2$ & $135.3 \pm 18.2$ & 0.462 \\
\hline DBP (mmHg) & $82.5 \pm 6.6$ & $83.3 \pm 8.4$ & $82.6 \pm 7.5$ & $84.6 \pm 9.6$ & $89.3 \pm 12.2$ & $92.8 \pm 13.8$ & 0.254 \\
\hline MAP (mmHg) & $96.5 \pm 8.3$ & $99.4 \pm 9.7$ & $97.4 \pm 9.0$ & $98.6 \pm 9.7$ & $103.2 \pm 10.3$ & $107.0 \pm 12.2$ & 0.294 \\
\hline HR (beats/min) & $60.9 \pm 7.5$ & $84.6 \pm 20.7$ & $84.4 \pm 10.2$ & $77.9 \pm 17.6$ & $71.4 \pm 14.9$ & $76.0 \pm 10.9$ & $<0.001$ \\
\hline $\mathrm{RR}$ (per min) & $13.7 \pm 2.4$ & $17.8 \pm 3.7$ & $16.3 \pm 3.1$ & $15.1 \pm 4.4$ & $15.5 \pm 4.3$ & $18.5 \pm 3.9$ & 0.016 \\
\hline $\mathrm{SpO}_{2}(\%)$ & $99.0 \pm 1.1$ & $87.1 \pm 6.2$ & $88.2 \pm 3.4$ & $89.8 \pm 3.9$ & $91.5 \pm 2.4$ & $92.0 \pm 3.0$ & $<0.001$ \\
\hline LLS & $0.0 \pm 0.0$ & $0.4 \pm 0.8$ & $1.0 \pm 1.5$ & $0.5 \pm 0.7$ & $0.4 \pm 0.5$ & $0.0 \pm 0.0$ & 0.164 \\
\hline
\end{tabular}

${ }^{a}$ Until 72 hours; LLS was analyzed using Friedman test.

ANOVA, analysis of variance; DBP, diastolic blood pressure; H, height; HR, heart rate; LLS, Lake Louise Score; MAP, mean arterial blood pressure; $\mathrm{R}$, resistance; $\mathrm{RR}$, respiratory rate; $\mathrm{SBP}$, systolic blood pressure; $\mathrm{SpO}_{2}$, oxygen saturation; $\mathrm{Xc}$, reactance; $\Delta \mathrm{VL}$ (\%), changes of vector length from baseline $(\%)$.

pressure, HR, RR, $\mathrm{SpO}_{2}$, and LLS are reported in Table 2. One subject developed AMS at 24 hours (LLS =5), but none of the subjects had a clinically relevant reduction in appetite, nausea, or vomiting during the study period. HR, RR, and $\mathrm{SpO}_{2}$ showed changes during the first 72 hours $(p<0.001$, $p=0.016$, and $p<0.001$, respectively). HR was higher at 9 and 24 hours compared to baseline $(p=0.022$ and $p=0.001$, respectively), while $\mathrm{SpO}_{2}$ remained lower for the whole period compared to baseline $(p<0.001$ for every time point until 72 hours, $p=0.005$ for 8 days).

At baseline, all subjects had their baseline impedance vectors within the $95 \%$ tolerance ellipse for a Caucasian reference population (Table 2) (Piccoli et al., 1995). The baseline impedance vector of five subjects was within the $50 \%$ tolerance ellipses, while three subjects fell between the $50 \%$ and $75 \%$ tolerance ellipses and three subjects between the $75 \%$ and $95 \%$ tolerance ellipses. In the R-Xc bivariate plane for body water status estimation (Fig. 2), the impedance vector showed a lengthening at 9 hours $(p=0.011)$, 48 hours $(p=0.033)$, and 72 hours $(p=0.015)$ compared to baseline (i.e., a relative TBW loss) (red ellipses). Between 9 and 24 hours (blue ellipse), there was a marginal shortening of impedance vector $(p=0.097)$ (i.e., subjects tended to demonstrate euhydration). However, the impedance vector displacement at 24 hours was not different from the baseline ( $p>0.05$; dashed ellipse). The impedance vector length on the eighth day was not significantly different from baseline or 72 hours (both $p>0.05$ ) (data not shown).

NT-proBNP values for all subjects remained within the range previously described in healthy subjects at baseline. $\ln$ (NT-proBNP) showed marginal changes during exposure to altitude within the first 72 hours $(p=0.082)$, with a peak after 24 hours ( $p=0.041$ compared with baseline) (Table 3$)$. $\ln (\mathrm{NT}-$ proBNP) values on the eighth day were not different from baseline $(p>0.05)$. In the majority of other peripheral venous blood and urine biochemical parameters, no significant changes were demonstrated during the period of stay at high altitude (Table 3). There was a trend to increase $\mathrm{Na}^{+}$excretion (i.e., natriuresis), specifically at 9 hours compared to baseline.

There was no difference between pre- and nonacclimatized subjects both for vector length ( $p=0.647$ for preacclimatization, $p=0.785$ for interaction of preacclimatization with time) and $\ln (\mathrm{NT}$-proBNP) $(p=0.377$ for preacclimatization, $p=0.141$ for interaction of preacclimatization with time) when comparing changes over time.

Correlation analysis (Table 4) showed that changes in $\ln \left(\mathrm{NT}\right.$-proBNP) were correlated with changes in serum $\mathrm{Na}^{+}$ $(p=0.002)$ and $\mathrm{Cl}^{-}(p=0.002)$, and marginally with serum $\mathrm{K}^{+}(p=0.068)$. There was no correlation between changes in vector length and $\ln (\mathrm{NT}-$ proBNP $)(\mathrm{p}=0.656)$.

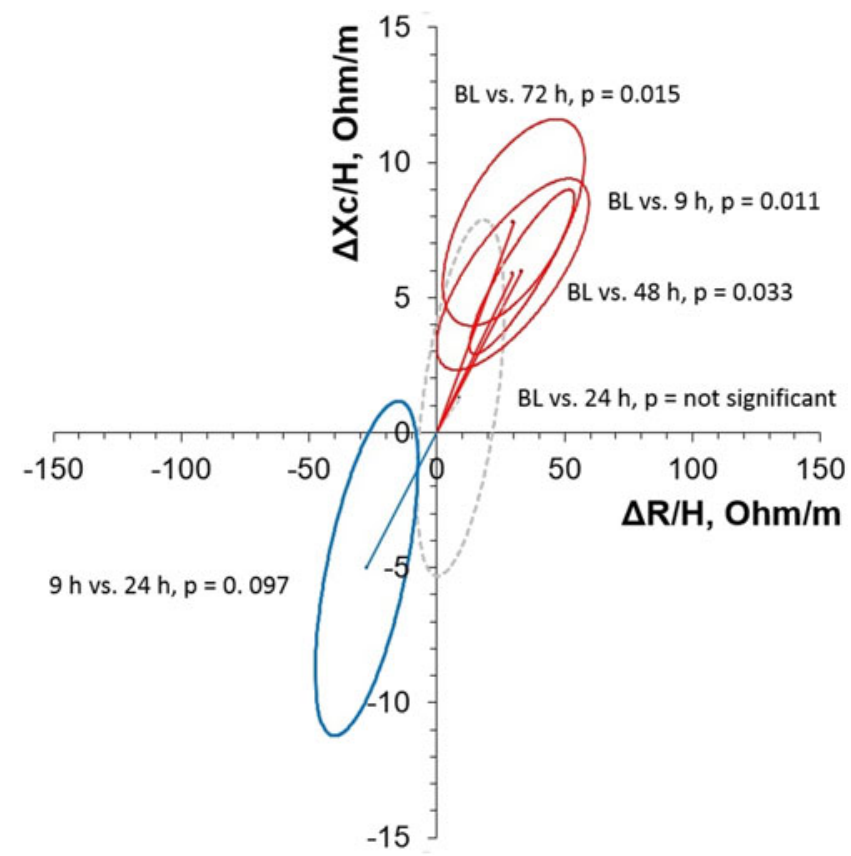

FIG. 2. The $95 \%$ confidence ellipses and the changes in vector length at different time points during altitude exposure compared to baseline. Red ellipses show a relative total body water loss compared to baseline, dashed ellipses a relative euhydrated status compared to baseline, and blue ellipses a relative total body water gain between two specific time points. $\Delta$, delta; $\mathrm{BL}$, baseline; $\mathrm{H}$, height; $\mathrm{R}$, resistance; $\mathrm{Xc}$, reactance. Color images are available online at www.liebertpub.com/ham 
Table 3. Peripheral Venous Blood and Urine Biochemical Parameters

\begin{tabular}{|c|c|c|c|c|c|c|}
\hline $\begin{array}{l}\text { Biochemical } \\
\text { parameters }\end{array}$ & Baseline & 9 hours & 24 hours & 72 hours & 8 days & $\begin{array}{c}\text { Repeated } \\
\text { measures ANOVA } \\
\text { p-value }\end{array}$ \\
\hline \multicolumn{7}{|l|}{ Blood parameters } \\
\hline ln(NT-proBNP) & $3.0 \pm 0.8$ & $4.2 \pm 0.7$ & $4.5 \pm 1.1$ & $3.9 \pm 1.4$ & $2.9 \pm 0.9$ & 0.082 \\
\hline $\begin{array}{l}{[\mathrm{NT}-\text { proBNP }} \\
(\mathrm{pg} / \mathrm{mL})]\end{array}$ & {$[27.8 \pm 22.4]$} & {$[79.3 \pm 53.5]$} & {$[133.6 \pm 108.8]$} & {$[126.7 \pm 234.9]$} & {$[27.0 \pm 26.8]$} & \\
\hline Urea (mg/dL) & $37.4 \pm 9.0$ & $36.0 \pm 9.5$ & $37.7 \pm 9.0$ & $31.4 \pm 7.8$ & $41.0 \pm 6.1$ & 0.226 \\
\hline Creatinine (mg/dL) & $1.0 \pm 0.2$ & $1.1 \pm 0.2$ & $1.1 \pm 0.2$ & $1.0 \pm 0.2$ & $1.1 \pm 0.1$ & 0.332 \\
\hline $\mathrm{Na}^{+}(\mathrm{mmol} / \mathrm{L})$ & $140.3 \pm 1.4$ & $141.2 \pm 1.5$ & $140.9 \pm 1.1$ & $139.7 \pm 2.5$ & $139.2 \pm 3.2$ & 0.489 \\
\hline $\mathrm{K}^{+}(\mathrm{mmol} / \mathrm{L})$ & $4.2 \pm 0.3$ & $4.2 \pm 0.4$ & $4.0 \pm 0.2$ & $4.6 \pm 0.5$ & $4.3 \pm 0.4$ & 0.083 \\
\hline $\mathrm{Cl}-(\mathrm{mmol} / \mathrm{L})$ & $100.9 \pm 2.7$ & $103.5 \pm 1.9$ & $104.5 \pm 2.8$ & $103.4 \pm 3.0$ & $102.5 \pm 1.8$ & 0.058 \\
\hline $\begin{array}{l}\text { Osmolality } \\
\qquad(\mathrm{mosmol} / \mathrm{kg})\end{array}$ & $292.0 \pm 6.1$ & $292.7 \pm 3.7$ & $288.8 \pm 3.4$ & $289.4 \pm 4.4$ & $289.3 \pm 4.0$ & 0.244 \\
\hline \multicolumn{7}{|l|}{ Urine parameters } \\
\hline $\mathrm{Na}^{+}(\mathrm{mmol} / \mathrm{L})$ & $126.1 \pm 45.2$ & $159.1 \pm 60.4$ & $122.3 \pm 58.2$ & $130.6 \pm 55.8$ & $145.2 \pm 64.6$ & 0.695 \\
\hline $\mathrm{K}^{+}(\mathrm{mmol} / \mathrm{L})$ & $60.7 \pm 25.1$ & $46.7 \pm 26.2$ & $44.4 \pm 22.9$ & $39.4 \pm 23.2$ & $67.5 \pm 30.3$ & 0.168 \\
\hline $\mathrm{Cl}^{-}(\mathrm{mmol} / \mathrm{L})$ & $159.8 \pm 63.4$ & $153.0 \pm 63.2$ & $116.3 \pm 71.0$ & $120.5 \pm 59.7$ & $153.5 \pm 73.3$ & 0.474 \\
\hline $\begin{array}{l}\text { Osmolality } \\
\text { (mosmol/kg) }\end{array}$ & $693.5 \pm 235.4$ & $760.0 \pm 273.9$ & $649.7 \pm 279.9$ & $575.9 \pm 242.1$ & $902.5 \pm 159.0$ & 0.452 \\
\hline
\end{tabular}

The NT-proBNP values in square bracket represent the actual values before $\ln$ transformation.

${ }^{a}$ Repeated measures ANOVA was carried out until 72 hours.

$\ln$, natural logarithm; NT-proBNP, N-terminal pro-B-type natriuretic peptide.

\section{Discussion}

For the first time, we report the fluctuation of TBW status in relation to NT-proBNP, both within hours of rapid exposure to $3830 \mathrm{~m}$ and up to 8 days of continuous altitude exposure. Body water status was monitored serially using BIA over the course of 8 days at moderate high altitude among healthy Caucasian subjects and subsequently analyzed with an R-Xc bivariate plane graph. All subjects resided at the Mt. Ortler test site for the duration of the study, without performing any undue physical exertion.

Table 4. Correlation of Changes in NT-ProBNP and Vector Length With Changes in Other Parameters Until 72 Hours OF High Altitude Exposure

\begin{tabular}{|c|c|c|c|c|}
\hline \multirow[b]{2}{*}{ Parameter } & \multicolumn{2}{|c|}{$\ln (N T-p r o B N P)$} & \multicolumn{2}{|c|}{$\begin{array}{c}\text { Vector } \\
\text { length }(\Omega / m)\end{array}$} \\
\hline & Correlation & $\mathrm{p}$ & Correlation & $\mathrm{p}$ \\
\hline $\mathrm{R} / \mathrm{H}(\Omega / \mathrm{m})$ & -0.099 & 0.662 & - & - \\
\hline $\mathrm{Xc} / \mathrm{H}(\Omega / \mathrm{m})$ & -0.197 & 0.379 & - & - \\
\hline Vector length $(\Omega / m)$ & -0.101 & 0.656 & - & - \\
\hline Phase angle $\left({ }^{\circ}\right)$ & -0.299 & 0.176 & - & - \\
\hline Urea, blood (mg/dL) & 0.227 & 0.297 & -0.303 & 0.133 \\
\hline $\begin{array}{l}\text { Creatinine, blood } \\
(\mathrm{mg} / \mathrm{dL})\end{array}$ & 0.122 & 0.579 & 0.041 & 0.841 \\
\hline $\mathrm{Na}^{+}$, blood $(\mathrm{mmol} / \mathrm{L})$ & 0.623 & 0.002 & -0.338 & 0.091 \\
\hline $\mathrm{K}^{+}$, blood $(\mathrm{mmol} / \mathrm{L})$ & -0.396 & 0.068 & 0.163 & 0.436 \\
\hline $\mathrm{Cl}^{-}$, blood $(\mathrm{mmol} / \mathrm{L})$ & 0.616 & 0.002 & 0.035 & 0.867 \\
\hline $\begin{array}{l}\text { Osmolality, blood } \\
\text { (mosmol/kg) }\end{array}$ & -0.262 & 0.227 & 0.046 & 0.824 \\
\hline $\mathrm{Na}^{+}$, urine $(\mathrm{mmol} / \mathrm{L})$ & 0.229 & 0.293 & 0.037 & 0.848 \\
\hline $\mathrm{K}^{+}$, urine $(\mathrm{mmol} / \mathrm{L})$ & -0.192 & 0.379 & -0.346 & 0.061 \\
\hline $\mathrm{Cl}^{-}$, urine $(\mathrm{mmol} / \mathrm{L})$ & -0.095 & 0.666 & -0.215 & 0.254 \\
\hline $\begin{array}{l}\text { Osmolality, urine } \\
\quad(\mathrm{mosmol} / \mathrm{kg})\end{array}$ & 0.102 & 0.644 & -0.167 & 0.378 \\
\hline
\end{tabular}

NT-proBNP, N-terminal pro-B-type natriuretic peptide; $p$, p-value.
Acute fluid loss was demonstrated within the first 9 hours, after which a switch to active euhydration was suggested up to 24 hours. Subsequently, compared to baseline, a progressive dehydration between 48 and 72 hours was shown. However, BIVA measurements taken at the eighth day were not significantly different from baseline. There was a significant increase of NT-proBNP after ascent with a peak at 24 hours, although similarly to BIVA values, NT-proBNP returned to baseline after 8 days of altitude exposure.

Within this study, changes in the impedance vector length (illustrated with $95 \%$ confidence ellipses) reveal highly reactive TBW dynamics over the course of hours to days of continuous moderately high altitude exposure. Primarily, the subjects were relatively dehydrated (9 hours), despite a sufficient fluid intake. This physiological response has been previously described in humans after acute exposure (26 hours) to both hypobaric (equivalent to $6000 \mathrm{~m}$ ) (Koller et al., 1991) and normobaric (equivalent to $4000 \mathrm{~m}$ ) hypoxia (Swenson et al., 1995). Acute exposure to hypoxia leading to a rapid rise in HVR could be postulated to regulate fluid balance through multiple pathways. First, to correct respiratory alkalosis caused by elevated HVR, bicarbonaturia reportedly reduces blood $\mathrm{pH}$ to within an acidic range. Second, Swenson et al. (1995) suggested that HVR itself is sufficient to mediate diuresis irrespective of blood acid-base changes. Finally, Koller et al. (1991) previously suggested that increased urinary flow is associated with a concomitant increase in atrial natriuretic peptide, plus a slight suppression of arginine-vasopressin secretion; both of which are proposed to offset increased cardiac volume overload at altitude. Shortening of the impedance vector between 9 and 24 hours shows the tendency to retain fluid. This was an unexpected finding. The reported fluid gain from 9 to 24 hours could be attributable to a stabilization in ventilatory response during preliminary acclimatization, plus slightly depressed ventilatory drive during sleep (Pamenter and Powell, 2016), but no data are available in the current study to elucidate the reason. All 
subjects, apart from one who quickly recovered within 24 36 hours at high altitude, did not develop AMS. Therefore, the progressive dehydration reported at 48 and 72 hours can be assumed to simply represent the physiological response to continuous moderate high altitude exposure.

From a clinical perspective, an increased level of NTproBNP is closely associated with volume overload and renal and cardiac function impairments (Chung et al., 2008; David et al., 2008; Booth et al., 2010; Nongnuch et al., 2014). Fluid retention is correlated with NT-proBNP in chronic kidney patients (Yilmaz et al., 2014). High altitude hypoxia exerts tremendous stress on the heart (Holloway et al., 2011; Mellor et al., 2014) and represents by itself a stimulus for NTproBNP release (Casals et al., 2009; Woods et al., 2011a; Mellor et al., 2014). Hence, stress on the heart and kidneys may enhance NT-proBNP production, reflecting both fluid overload and stress related to hypoxia exposure. Studies performed at very high altitude $(>5000 \mathrm{~m})$ have reported a significant increase in BNP with altitude exposure (Feddersen et al., 2009; Woods et al., 2011a). Similar to these previous findings (Woods et al., 2011a), we observed an elevation in NT-proBNP with time at altitude, reaching a maximum value at 24 hours. The elevated values of NTproBNP strengthen the indication that there is increased stress on the heart, as suggested by the increase in HR, with acutely rapid high altitude exposure, whereas the lack of correlation between changes in BIVA measurements and changes in NT-proBNP can only elude to the complexity of the interplay between hypoxia-stimulated cardiovascular, renal, and endocrine responses resulting in TBW regulation.

Serum urea and creatinine levels did not differ significantly with altitude exposure across all observed time points. Serum and urine osmolality and ion concentrations did not change significantly. Serum osmolality showed a nonsignificant trend to decrease at 24 and 72 hours compared to baseline which does not comply with the apparent dehydration at BIVA measurements (TBW). It is unclear whether this remains within the normal range, the two techniques describe different measurements or because the method of BIVA is more sensitive to detect dehydration, within the so-called "appropriate dehydration" range (i.e., a vector lengthening of only $10 \%-15 \%$ ) as a process of physiological acclimatization (Piccoli et al., 1996). Nevertheless, both in this study and previous work from Piccoli et al. (1996) healthy subjects who do not develop AMS showed an average impedance vector lengthening less than 10\%-15\%. In comparison, dehydration (to a greater extent) is considered a determinate risk factor at altitude and could mimic symptoms of AMS (Imray et al., 2010). Hence, it is recommended that trekkers, hikers, and mountaineers actively manage fluid uptake during altitude expeditions (Luks et al., 2010). However, overhydration has also been reportedly associated with incidence and severity of AMS (Westerterp et al., 1996; Loeppky et al., 2005; Gatterer et al., 2013).

AMS, high-altitude cerebral edema, and high-altitude pulmonary edema pathophysiology involves organ-specific fluid accumulation. Recent studies demonstrated decreased cerebral white matter water mobility (including brain stem and cerebellum) as early as 2 hours at $12 \% \mathrm{O}_{2}$ normobaric hypoxia (Lawley et al., 2013). During 10 hours of normobaric hypoxic exposure, increased gray matter volume was demonstrated in parallel to movement of fluid from the vascular compartment to other areas of the brain, thus leading to an increase in the overall brain volume (i.e., increased intracranial pressure) (Lawley et al., 2014). Interstitial fluid accumulation (edema) manifests also in other body regions, for example, B-lines in the lungs with passive exposure (Strapazzon et al., 2015) and exertion (Pratali et al., 2010; Edsell et al., 2014). On examination, it is important to note that it is difficult with BIVA measurements to have specific fluid measurement within the brain and lungs. Hence, altitudespecific pathophysiological fluid status within these organs is difficult to estimate with this technique. Similarly, NTproBNP does not seem to reflect organ-specific fluid accumulation (Strapazzon et al., 2015).

\section{Limitations}

Despite having recruited 19 subjects, we ended up analyzing only 11 subjects for TBW status since many of them did not have BIVA data at the 9 hours time point due to logistical challenges related to a field study. Nevertheless, we have used more time points for observation and reduced potential confounding factors as much as possible. The subjects were, in fact, mostly resting or had minimal physical exertion with a standardized food and fluid intake regime with regular meals. The 24-hour urinary output and body weight were not monitored due to field limitations. Finally, the low incidence of AMS in our study may also be partially due to the preacclimatization of six subjects.

\section{Conclusion}

We report the fluctuation of TBW status in relation to NTproBNP during rapid altitude exposure $(3830 \mathrm{~m})$ and up to 8 days of continuous high altitude residence at the same altitude in healthy lowland subjects. Subjects first became dehydrated (9 hours) and subsequently tended to exhibit a short period of euhydration over the next 15 hours. However, they remained dehydrated at 48 and 72 hours of continuous exposure. NT-proBNP does not simply reflect the TBW status during acute high altitude exposure and its changes were not correlated with BIVA measurements. Results also suggest that around $2 \mathrm{~L}$ of fluid per day may be sufficient during acute rapid exposure to high altitude to maintain hydration homeostasis, but only with minimal physical activity. Further studies with BIVA and NT-proBNP measurements in relation to HRV, sympathetic response, body weight, and 24-hour urinary output in both subjects performing exercise and patients with high altitude illnesses are necessary to better elucidate the clinical significance of changes in TBW status during exposure to acute hypobaric hypoxia.

\section{Acknowledgments}

The authors thank AKERN SRL, Firenze, Italy, for lending the equipment during the study. The authors are grateful to their fellow researchers (Georg Hofer, MD and Stefan Platzgummer, MD) and staff from EURAC Institute of Mountain Emergency Medicine, Bolzano, Italy (Karla Balkenhol, MD and Michael Pohl, MD), who played a crucial role to make this field study successful and for article preparation (Rachel Turner, MPhil). Finally, the authors thank our study subjects for their consent, time, and compliance.

\section{Author Disclosure Statement}

No competing financial interests exist. 


\section{References}

Basnyat B, Lemaster J, and Litch JA. (1999). Everest or bust: A cross sectional, epidemiological study of acute mountain sickness at 4243 meters in the Himalayas. Aviat Space Environ Med 70:867-873.

Bland JM, and Altman DG. (1995). Calculating correlation coefficients with repeated observations: Part $1-$ Correlation within subjects. BMJ 310:446-446.

Booth J, Pinney J, and Davenport A. (2010). N-terminal proBNP - marker of cardiac dysfunction, fluid overload, or malnutrition in hemodialysis patients? Clin J Am Soc Nephrol 5:1036-1040.

Buffa R, Saragat B, Cabras S, Rinaldi AC, and Marini E. (2013). Accuracy of specific BIVA for the assessment of body composition in the United States population. PLoS One 8:e58533.

Casals G, Ros J, Sionis A, Davidson MM, Morales-Ruiz M, and Jimenez W. (2009). Hypoxia induces B-type natriuretic peptide release in cell lines derived from human cardiomyocytes. Am J Physiol Heart Circ Physiol 297:H550-H555.

Castellani JW, Muza SR, Cheuvront SN, Sils IV, Fulco CS, Kenefick RW, Beidleman BA, and Sawka MN. (2010). Effect of hypohydration and altitude exposure on aerobic exercise performance and acute mountain sickness. J Appl Physiol (1985) 109:1792-1800.

Chung JH, Yun NR, Ahn CY, Lee WS, and Kim HL. (2008). Relationship between serum N-terminal pro-brain natriuretic peptide level and left ventricular dysfunction and extracellular water in continuous ambulatory peritoneal dialysis patients. Electrolyte Blood Press 6:15-21.

David S, Kumpers P, Seidler V, Biertz F, Haller H, and Fliser D. (2008). Diagnostic value of N-terminal pro-B-type natriuretic peptide (NT-proBNP) for left ventricular dysfunction in patients with chronic kidney disease stage 5 on haemodialysis. Nephrol Dial Transplant 23:1370-1377.

Edsell ME, Wimalasena YH, Malein WL, Ashdown KM, Gallagher CA, Imray CH, Wright AD, and Myers SD. (2014). High-intensity intermittent exercise increases pulmonary interstitial edema at altitude but not at simulated altitude. Wilderness Environ Med 25:409-415.

Ermolao A, Bergamin M, Rossi AC, Dalle Carbonare L, Zaccaria M. (2011). Cardiopulmonary response and body composition changes after prolonged high altitude exposure in women. High Alt Med Biol 12:357-369.

Feddersen B, Ausserer H, Haditsch B, Frisch H, Noachtar S, and Straube A. (2009). Brain natriuretic peptide at altitude: Relationship to diuresis, natriuresis, and mountain sickness. Aviat Space Environ Med 80:108-111.

Fulco CS, Hoyt RW, Baker-Fulco CJ, Gonzalez J, and Cymerman A. (1992). Use of bioelectrical impedance to assess body composition changes at high altitude. J Appl Physiol (1985) 72:2181-2187.

Gatterer H, Wille M, Faulhaber M, Lukaski H, Melmer A, Ebenbichler C, and Burtscher M. (2013). Association between body water status and acute mountain sickness. PLoS One 8:e73185.

Hackett PH, and Rennie D. (1979). Rales, peripheral edema, retinal hemorrhage and acute mountain sickness. Am J Med 67:214-218.

Hackett PH, Rennie D, Grover RF, and Reeves JT. (1981). Acute mountain sickness and the edemas of high altitude: A common pathogenesis? Respir Physiol 46:383-390.

Holloway CJ, Montgomery HE, Murray AJ, Cochlin LE, Codreanu I, Hopwood N, Johnson AW, Rider OJ, Levett DZ,
Tyler DJ, et al. (2011). Cardiac response to hypobaric hypoxia: Persistent changes in cardiac mass, function, and energy metabolism after a trek to Mt. Everest Base Camp. FASEB J 25:792-796.

Imray C, Wright A, Subudhi A, and Roach R. (2010). Acute mountain sickness: Pathophysiology, prevention, and treatment. Prog Cardiovasc Dis 52:467-484.

Koller EA, Buhrer A, Felder L, Schopen M, and Vallotton MB. (1991). Altitude diuresis: Endocrine and renal responses to acute hypoxia of acclimatized and non-acclimatized subjects. Eur J Appl Physiol Occup Physiol 62:228-234.

Lawley JS, Alperin N, Bagci AM, Lee SH, Mullins PG, Oliver SJ, and Macdonald JH. (2014). Normobaric hypoxia and symptoms of acute mountain sickness: Elevated brain volume and intracranial hypertension. Ann Neurol 75:890-898.

Lawley JS, Oliver SJ, Mullins PG, and Macdonald JH. (2013). Investigation of whole-brain white matter identifies altered water mobility in the pathogenesis of high-altitude headache. J Cereb Blood Flow Metab 33:1286-1294.

Leaf DE, and Goldfarb DS. (2007). Mechanisms of action of acetazolamide in the prophylaxis and treatment of acute mountain sickness. J Appl Physiol (1985) 102:1313-1322.

Loeppky JA, Icenogle MV, Maes D, Riboni K, HinghoferSzalkay H, and Roach RC. (2005). Early fluid retention and severe acute mountain sickness. J Appl Physiol (1985) 98: 591-597.

Lukaski HC, Hall CB, and Siders WA. (2007). Assessment of change in hydration in women during pregnancy and postpartum with bioelectrical impedance vectors. Nutrition 23: 543-550.

Lukaski HC, and Piccoli A. (2012). Bioelectrical impedance vector analysis for assessment of hydration in physiological states and clinical conditions. In Handbook of Anthropometry: Physical Measures of Human Form in Health and Disease. RV Preedy, ed. Springer, New York. pp 287-305.

Luks AM, McIntosh SE, Grissom CK, Auerbach PS, Rodway GW, Schoene RB, Zafren K, and Hackett PH. (2010). Wilderness Medical Society consensus guidelines for the prevention and treatment of acute altitude illness. Wilderness Environ Med 21:146-155.

Mellor A, Boos C, Holdsworth D, Begley J, Hall D, Lumley A, Burnett A, Hawkins A, O'Hara J, Ball S, et al. (2014). Cardiac biomarkers at high altitude. High Alt Med Biol 15:452458.

Nerin MA, Palop J, Montano JA, Morandeira JR, and Vazquez M. (2006). Acute mountain sickness: Influence of fluid intake. Wilderness Environ Med 17:215-220.

Nongnuch A, Panorchan K, and Davenport A. (2014). Predialysis NTproBNP predicts magnitude of extracellular volume overload in haemodialysis patients. Am J Nephrol 40: 251-257.

Norris JN, Viirre E, Aralis H, Sracic MK, Thomas D, and Gertsch JH. (2012). High altitude headache and acute mountain sickness at moderate elevations in a military population during battalion-level training exercises. Mil Med 177: 917-923.

Pamenter ME, and Powell FL. (2016). Time domains of the hypoxic ventilatory response and their molecular basis. Compr Physiol 6:1345-1385.

Piccoli A. (2010). Bioelectric impedance measurement for fluid status assessment. Contrib Nephrol 164:143-152.

Piccoli A, Nigrelli S, Caberlotto A, Bottazzo S, Rossi B, Pillon L, and Maggiore Q. (1995). Bivariate normal values of the bioelectrical impedance vector in adult and elderly populations. Am J Clin Nutr 61:269-270. 
Piccoli A, Piazza P, Noventa D, Pillon L, and Zaccaria M. (1996). A new method for monitoring hydration at high altitude by bioimpedance analysis. Med Sci Sports Exerc 28:1517-1522.

Piccoli A, Rossi B, Pillon L, and Bucciante G. (1994). A new method for monitoring body fluid variation by bioimpedance analysis: The RXc graph. Kidney Int 46:534-539.

Pollastri L, Lanfranconi F, Tredici G, Schenk K, Burtscher M, and Gatterer H. (2016). Body fluid status and physical demand during the Giro d'Italia. Res Sports Med 24:30-38.

Pratali L, Cavana M, Sicari R, and Picano E. (2010). Frequent subclinical high-altitude pulmonary edema detected by chest sonography as ultrasound lung comets in recreational climbers. Crit Care Med 38:1818-1823.

Richardson A, Watt P, and Maxwell N. (2009a). The effect of hypohydration severity on the physiological, psychological and renal hormonal responses to hypoxic exercise. Eur J Appl Physiol 106:123-130.

Richardson A, Watt P, and Maxwell N. (2009b). Hydration and the physiological responses to acute normobaric hypoxia. Wilderness Environ Med 20:212-220.

Strapazzon G, Brugger H, Dal Cappello T, Procter E, Hofer G, and Lochner P. (2014). Factors associated with optic nerve sheath diameter during exposure to hypobaric hypoxia. Neurology 82:1914-1918.

Strapazzon G, Vezzaro R, Hofer G, Dal Cappello T, Procter E, Balkenhol K, Platzgummer S, and Brugger H. (2015). Factors associated with B-lines after exposure to hypobaric hypoxia. Eur Heart J Cardiovasc Imaging 16:1241-1246.

Swenson ER, Duncan TB, Goldberg SV, Ramirez G, Ahmad S, and Schoene RB. (1995). Diuretic effect of acute hypoxia in humans: Relationship to hypoxic ventilatory responsiveness and renal hormones. J Appl Physiol (1985) 78:377-383.

Weber M, and Hamm C. (2006). Role of B-type natriuretic peptide (BNP) and NT-proBNP in clinical routine. Heart 92: 843-849.
West JB. (2004). The physiologic basis of high-altitude diseases. Ann Intern Med 141:789-800.

Westerterp KR, Robach P, Wouters L, and Richalet JP. (1996). Water balance and acute mountain sickness before and after arrival at high altitude of 4,350 m. J Appl Physiol (1985) 80: 1968-1972.

Woods DR, Begley J, Stacey M, Smith C, Boos CJ, Hooper T, Hawkins A, Hodkinson P, Green N, and Mellor A. (2012). Severe acute mountain sickness, brain natriuretic peptide and NT-proBNP in humans. Acta Physiol (Oxf) 205:349-355.

Woods DR, Hooper T, Hodkinson P, Ball S, Wakeford R, Peaston B, Bairsto C, Green N, and Mellor A. (2011a). Effects of altitude exposure on brain natriuretic peptide in humans. Eur J Appl Physiol 111:2687-2693.

World Medical Association. (2013). World Medical Association Declaration of Helsinki: Ethical principles for medical research involving human subjects. JAMA 310:2191-2194.

Yilmaz Z, Yildirim Y, Oto F, Aydin FY, Aydin E, Kadiroglu AK, and Yilmaz ME. (2014). Evaluation of volume overload by bioelectrical impedance analysis, NT-proBNP and inferior vena cava diameter in patients with stage $3 \& 4$ and 5 chronic kidney disease. Ren Fail 36:495-501.

Address correspondence to:

Giacomo Strapazzon, $M D, P h D$

EURAC Institute of Mountain Emergency Medicine

Viale Druso 1

39100 Bolzano Italy

E-mail: giacomo.strapazzon@eurac.edu

Received April 25, 2017; accepted in final form August 7, 2017. 